\title{
The 17 March 2015 storm: the associated magnetic flux rope structure and the storm development
}

\author{
Katsuhide Marubashi ${ }^{1,2,3^{*}}$ (D), Kyung-Suk Cho ${ }^{1,4}$, Rok-Soon Kim ${ }^{1,4}$, Sujin Kim¹, Sung-Hong Park ${ }^{5}$ \\ and Hiromitsu Ishibashi ${ }^{3}$
}

\begin{abstract}
The objective of this study is (1) to determine the magnetic cloud (MC) structure associated with the 17 March 2015 storm and (2) to gain an insight into how the storm developed responding to the solar wind conditions. First, we search MC geometries which can explain the observed solar wind magnetic fields by fitting to both cylindrical and toroidal flux rope models. Then, we examine how the resultant MC geometries can be connected to the solar source region to find out the most plausible model for the observed $\mathrm{MC}$. We conclude that the observations are most consistently explained by a toroidal flux rope with the torus plane nearly parallel to the ecliptic plane. It is emphasized that the observations are characterized by the peculiar spacecraft crossing through the $\mathrm{MC}$, in that the magnetic fields to be observed are southward throughout the passage. For understanding of the storm development, we first estimate the injection rate of the storm ring current from the observed Dst variation. Then, we derive an expression to calculate the estimated injection rate from the observed solar wind variations. The point of the method is to evaluate the injection rate by the convolution of the dawn-to-dusk electric field in the solar wind and a response function. By using the optimum response function thus determined, we obtain a modeled Dst variation from the solar wind data, which is in good agreement with the observed Dst variation. The agreement supports the validity of our method to derive an expression for the ring current injection rate as a function of the solar wind variation.
\end{abstract}

Keywords: Magnetic cloud, Coronal mass ejection, Flux rope, Solar wind, Solar wind magnetic field, Geomagnetic storm, Ring current, Injection rate, Dst

\section{Introduction}

The 17 March 2015 geomagnetic storm is the largest in Solar Cycle 24 so far with minimum Dst of $-223 \mathrm{nT}$ and has stimulated many research activities (Kamide and Kusano 2015; Kataoka et al. 2015; Liu et al. 2015; Cho et al. 2016; Wang et al. 2016). The large geomagnetic storm was somewhat surprising, and space weather agencies worldwide failed to predict that such a severe storm would occur (Kamide and Kusano 2015; Kataoka et al. 2015, Wang et al. 2016). Thus, the prior concern was mechanisms having caused such an intense storm. While some particular ideas have been proposed such

\footnotetext{
*Correspondence: kmaru_bg@kzh.biglobe.ne.jp

${ }^{1}$ Korea Astronomy and Space Science Institute, Daejeon 305-348, Korea Full list of author information is available at the end of the article
}

as the superposition effect of two successive storms as evidenced by the two-step main phase (Kamide and Kusano 2015), and intensification of magnetic fields and plasma densities in the interplanetary coronal mass ejection (ICME) due to a possible pileup effect (Kataoka et al. 2015), the main concern of the remaining studies is to explain mechanisms to create the solar wind conditions that caused such an intense storm.

It is commonly accepted in the foregoing studies that a magnetic cloud (MC) is involved which originated from a coronal mass ejection (CME) on 15 March 2015 from AR 12297 and arrived at $1 \mathrm{AU}$ on 17 March. As for the MC structure, however, several different geometries are being proposed. One reason is the complexity in the solar wind data due to superposed disturbances which prevents easy determination of boundaries for this particular 
MC. In addition, the fitting analyses for determining the MC structure yield different geometries depending on MC models used in the fitting. It is also true that the fitting analysis does not always assure the uniqueness of the solution for the MC structure (Marubashi and Cho 2015). Thus, careful analyses are needed to obtain an optimum interpretation about the global geometry of the 17 March 2015 MC.

This paper is aimed at answering two questions: (1) what MC structure provides the most reasonable interpretation for the observed magnetic field variation, and (2) how the observed solar wind conditions are connected to the development of the intense storm. For this purpose, we first search for flux rope geometries, which can explain the observed MC and select the most probable one by considering the link to its solar source. Then, we examine the Dst development as a response to the observed solar wind conditions based on the model originally proposed by Burton et al. (1975).

\section{MC structure and its connection to the solar source event}

Figure 1 shows the solar wind characteristics for 17-18 March 2015 obtained from the WIND spacecraft. Plotted are from the top, magnetic field intensity, the $X, Y$, and $Z$ components in geocentric solar ecliptic (GSE) coordinates, degree of magnetic field fluctuations (defined by standard deviations divided by mean values of the field vector data of higher time resolution), solar wind speed, number density, the number density ratio of $\mathrm{He}^{++}$to $\mathrm{H}^{+}$, proton temperature together with temperature expected statistically from the solar wind speed (dashed curve) (Lopez 1987), and the plasma beta based on proton. The bottom diagram shows magnetic field vectors projected on the $X Y, X Z$, and $Y Z$ planes. The red curves in the panels $1-4$ and 5 present model values obtained from the optimal torus model as described below.

We can see MC-like structure(s) around the time interval indicated by two vertical solid lines and a preceding shock at 04:00 UT (dashed line). Our first task is to select the appropriate $\mathrm{MC}$ boundaries and determine the structure by model fitting. The MC boundaries are generally selected by identifying regions characterized by enhanced magnetic field intensity, smooth magnetic field rotation, low proton temperature, low plasma beta, bidirectional superthermal electron flow, etc. though it is often the case that those regions do not coincide with each other (e.g., Gosling 1990; Neugebauer and Gosling 1997; Jian et al. 2006). Four different ideas have been proposed for the MC boundaries by recent studies. They are: (i) 17/13:00-18/09:00 (Gopalswamy et al. 2015), (ii) 17/11:00-17/23:10 (Cho et al. 2016), (iii) 17/13:05-17/23:20 (Kataoka et al. 2015; Wang et al. 2016), and (iv) two MCs, 17/09:00-17/18:00 and 17/18:00-18/17:00 (Liu et al. 2015). (Note that time intervals indicated above for selection by Gopalswamy et al. (2015) and Kataoka et al. (2015) are different from those in their original paper, because their corresponding times were taken from OMNI data, in which time is shifted for the solar wind transit from the observing satellite to the nose of Earth's bow shock.) From the viewpoint of MC modeling by flux rope models, it seems that the selections (i) and (iv) are inappropriate. For (i), the rapid change in the magnetic field direction around 17/23:20 looks more like a boundary between two plasma regimes of different characteristics. For (iv), there is no strong reasoning for cutting the structure into two separate parts at 17/18:00, where field changes are rather smooth and continuous.

We see in Fig. 1 that the magnetic field rotation in the $Y Z$ plane should be rather smooth, if the large amplitude fluctuations from 14:10 to 16: 50 UT (indicated by two dash-dot lines) on 17 March are disregarded. We compared variations in velocity vectors and in magnetic field vectors throughout the possible $\mathrm{MC}$ intervals and found that they are well correlated with each other during the above time interval. This suggests that those fluctuations are due to superposition of small-scale disturbances of Alfvénic nature on the flux rope similarly to phenomena reported by Gosling et al. (2010). Figure 2 shows a correlation diagram between $\Delta B x, \Delta B y$, $\Delta B z$ and $\Delta V x, \Delta V y, \Delta V z$ (differences from time averages, $B_{0}$ and $V_{0}$, over the time interval 14:10-16:50 UT on 17 March). Ideally, the relation between the wave components of magnetic field $\Delta B x, y, z$ and velocity $\Delta V x, y, z$ is given by

$$
\Delta V x, y, z=\left(V_{\mathrm{A}} / B_{0}\right) \cdot \Delta B x, y, z
$$

where $V_{\mathrm{A}}$ is the Alfvén velocity in the background magnetic field $B_{0}$. As a whole, the correlation coefficient is 0.74 , and we obtained the relation: $\Delta V=0.49\left(V_{\mathrm{A}} / B_{0}\right) \cdot \Delta B$.

Figure 3 presents the result of minimum variance analysis (Sonnerup and Cahill 1967) for the time interval from 11:00 to 23:10 UT on 17 March with 1-min averaged data (14:10-16:50 UT data being excluded), where $B_{1}, B_{2}$ and $B_{3}$ denote the magnetic field components in the maximum, intermediate and minimum variance directions, respectively. The starting point of the hodograph is indicated by a circle. The directions $(\phi, \theta)$, longitude and latitude, of three principal axes and the corresponding eigenvalues $(\lambda)$ are: $\left(\oint_{1}, \theta_{1}\right)=\left(271.6^{\circ}\right.$, $\left.15.2^{\circ}\right), \lambda_{1}=191.4 ;\left(\phi_{2}, \theta_{2}\right)=\left(215.9^{\circ},-64.3^{\circ}\right), \lambda_{2}=29.3$; $\left(g_{3}, \theta_{3}\right)=\left(355.8^{\circ},-20.2^{\circ}\right), \lambda_{3}=6.4$. A clear anticlockwise 


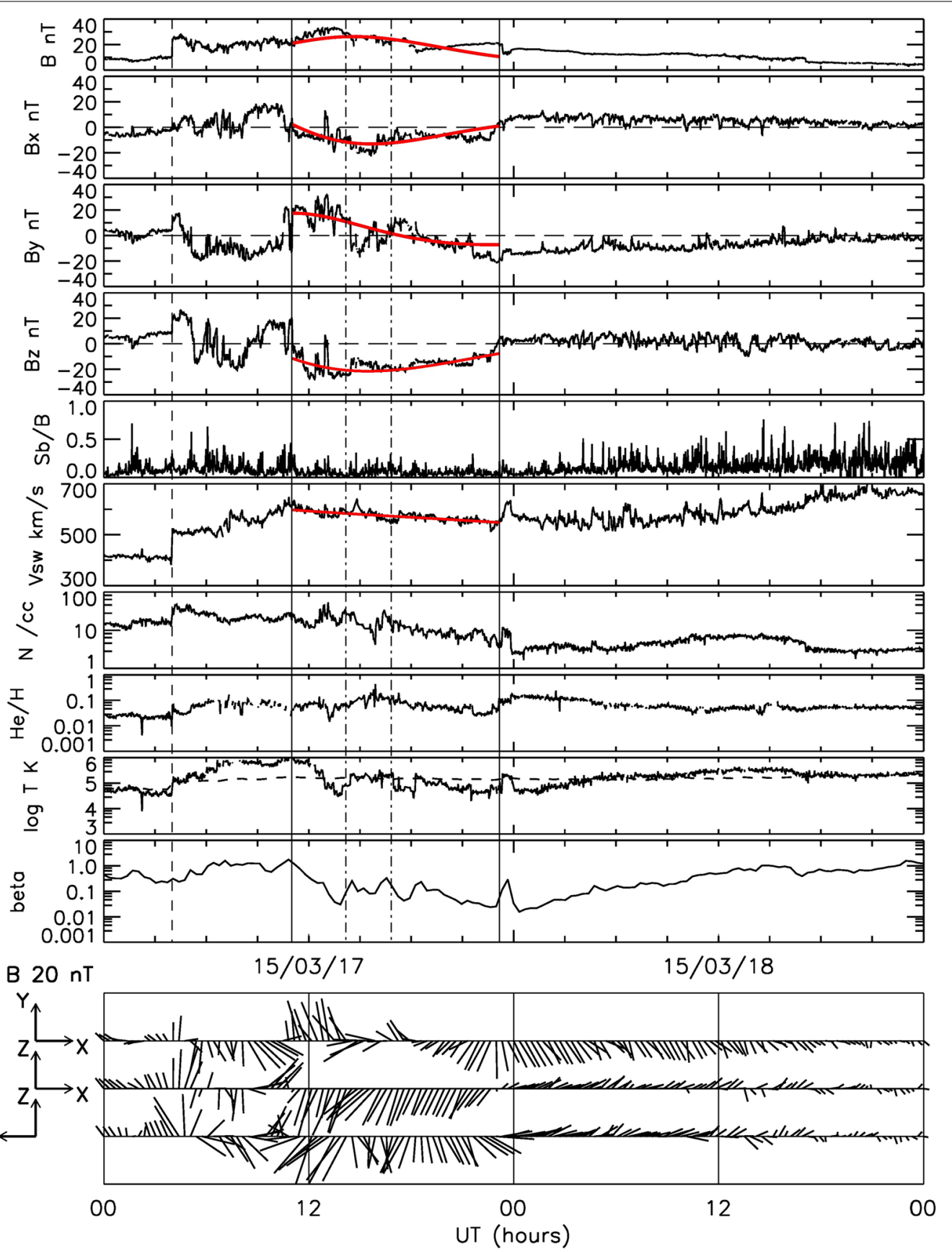

Fig. 1 Solar wind conditions from WIND as the background of the 17 March 2015 storm. The upper diagram shows variations in the magnetic field intensity $(B)$, three components ( $B x, B y$, and $B z$ ), degree of fluctuations ( $r m s$ divided by average $B$ ), solar wind speed, proton number density, the number density ratio of $\mathrm{He}^{++}$to proton, the proton temperature, and plasma beta based on protons. The lower is the vector diagram showing projections onto $X-Y, X-Z$, and $Y-Z$ planes of the 20 -minute averaged magnetic field vectors. The magnetic cloud interval is indicated by two vertical solid lines. The dashed line marks the shock arrival time. The red curves depict the optimum result of fitting with a toroidal flux rope model

rotation is seen in a plane nearly perpendicular to the $Y Z$ plane as a whole, with occasional back-and-forth changes in direction. This rotation is also seen in the vector plot in Fig. 1.
We attempted to determine the magnetic field structure for selected time intervals by fitting to the flux rope models with cylindrical and toroidal shapes developed by Marubashi and Lepping (2007). The models are 


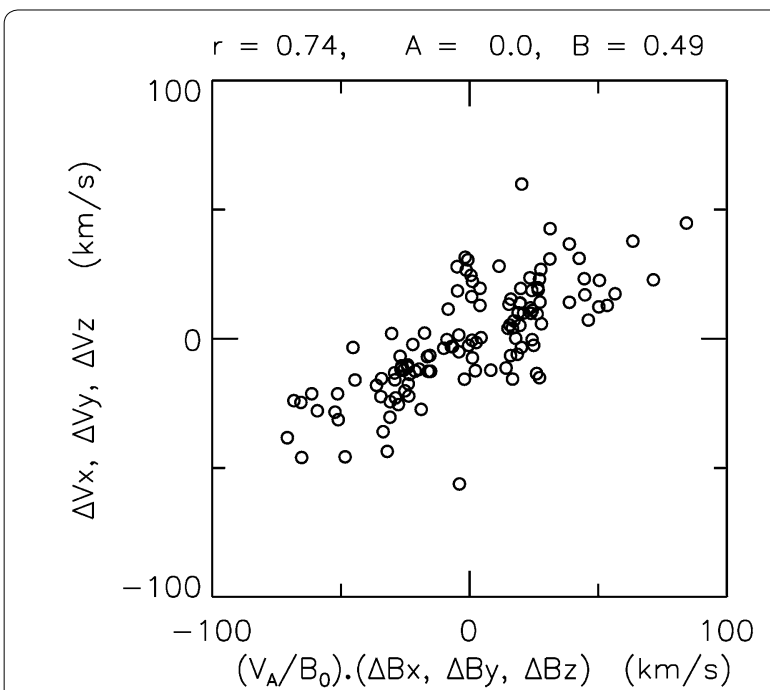

Fig. 2 A correlation diagram between $\Delta B x, \Delta B y, \Delta B z$ and $\Delta V x, \Delta V y$, $\Delta V z$ for the time interval 14:10-16:50 UT on 17 March. The correlation suggests that Alfvénic fluctuations are superposed during that time interval
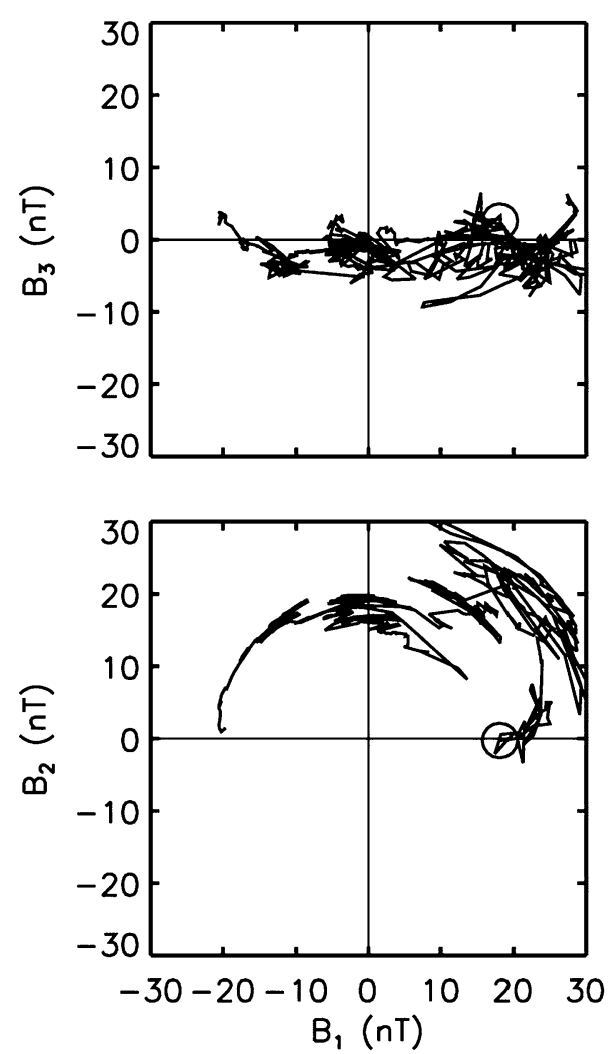

Fig. 3 A hodograph presentation of the minimum variance analysis for the 17 March 2015 magnetic cloud (MC). The MC interval is 11:00-23:10 UT, and the start point is indicated by a circle force-free flux rope models including the effect of selfsimilar expansion. The fitting was performed for intervals (ii) and (iii), with intention to see, if possible, which interval identifies more appropriate MC boundaries. In addition, we tried two data sets for each of the two intervals. One data set includes all observed data points within the MC intervals, and the other data set excludes data points corresponding to the Aflvénic fluctuations (14:10-16:50 UT). Thus, the fitting analysis was executed with four different data sets. As a result, we obtained one cylindrical flux rope model with right-handed chirality for each of the four data sets. The toroidal model fitting yielded one toroidal flux rope with right-handed chirality for each of the four data sets. In addition, three toroidal flux ropes with left-handed chirality were obtained from three data sets. (The details of the fitting results are described in Additional file 1: Appendix).

We try to determine which is the most plausible geometry for the observed MC out of the four cylinder models and seven torus models, by examining possible connection to the solar source. The foregoing studies (Gopalswamy et al. 2015; Kataoka et al. 2015; Liu et al. 2015; Cho et al. 2016; Wang et al. 2016) agree that a halo CME associated with a C9.1 flare which started at 01:15 UT on 15 March in AR 12297 (S22W25) was the solar source event of the MC. The general agreement comes from the fact that the above CME is the most prominent event and satisfies the consideration about the transit time from the Sun to $1 \mathrm{AU}$.

Figure 4 presents (a) a difference image of the CME at 05:30:05 UT on 15 March from the Solar Heliospheric Observatory/Large Angle and Spectrometric Coronagraph C3 (SOHO/LASCO-C3), (b) a solar image of 304 A obtained by the Solar Dynamics Observatory/Atmospheric Imaging Assembly (SDO/AIA) at 01:38:44 UT, and (c) a magnetograph at 01:00 UT from the SDO/Helioseismic and Magnetic Imager (HMI). In Fig. 4a, the yellow line shows the approximate envelop of the halo CME, which first appeared at 01:48 UT in the field of view of LASCO C2. The AIA 304 image in Fig. 4b clearly shows two ribbons associated with the post-eruption arcade of the C9.1 flare as indicated by the yellow oval that can be taken as the source event of the CME of concern and the interplanetary magnetic flux rope of 17 March. The yellow vector in Fig. 4c shows the approximate position of the polarity inversion line (PIL) corresponding to the two ribbons in the AIA 304 image. The orientation of the arrow was determined so that the orientation coincides with the direction of sheared field component along the PIL corresponding to the right-handed helicity as evidenced by the $\mathrm{S}$-shaped eruptive filament. Wang et al. (2016) analyzed the source signatures of this CME and 
a

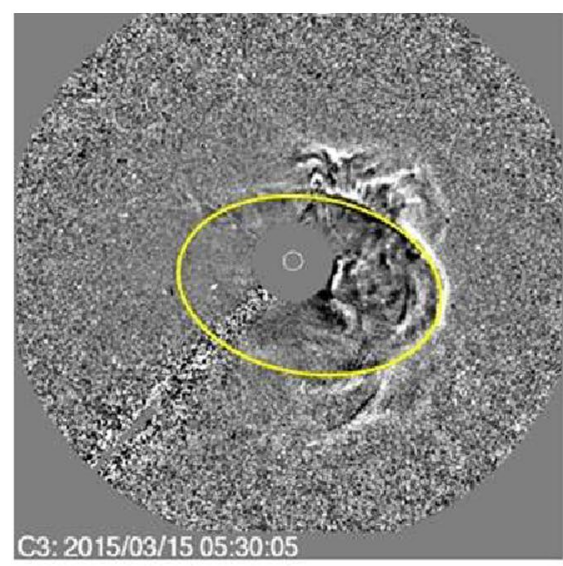

C3: 2015/03/15 05:30:05 b

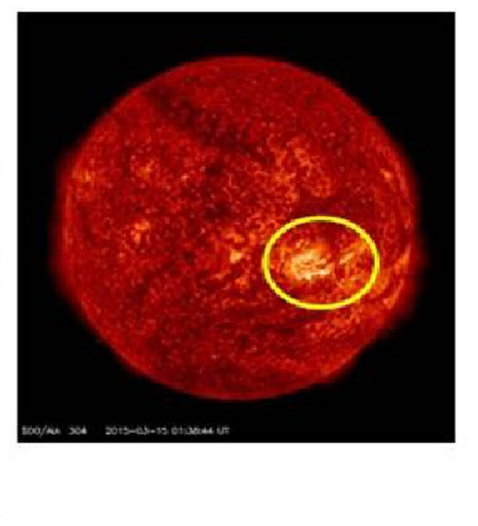

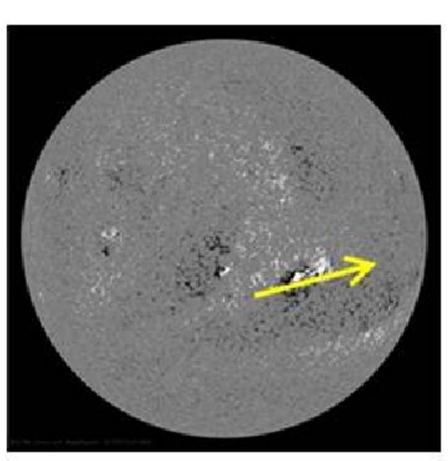

SDO/HMI 03/15 01:00

Fig. 4 Images connected to the solar source event of the 17 March 2015 MC. a A difference image of the halo CME at 05:30:05 UT from the SOHO/ LASCO C3. The yellow oval depicts the envelope of the CME. b An image from SDO/AIA 304 telescope at 01:38:44 UT. Two ribbons are clearly seen, which is related to the PEA. c A magnetograph from the SDO/HMI at 01:00 UT, white positive, black negative. The yellow arrow indicates the orientation of magnetic field component along the PIL

showed that the erupted flux rope has its axis parallel to the PIL. Then, it is obvious from the CME triggering theory (e.g., Kusano et al. 2012) that the flux rope should have the axial field parallel to the yellow arrow and the perpendicular field directed southward on the front side. Thus, the flux rope created in the corona should have the right-handed chirality with the axis directed along the yellow vector. Therefore, it is clear that three torus models with left-handed chirality (La-2, Lb-2, Sb-2 in Additional file 1: Figure A1 in Appendix) is inappropriate for explaining the observed MC. In addition, the position of spacecraft crossing for each of the three looks inconsistent with the source position of S22W25. They are all crossed by the spacecraft near the western end.

The consideration of the solar source position further suggests that other two geometries (Sa-1, Sb-1) are not appropriate for the model of the observed MC. In these two cases, it is required that the main body of the MC extends on the northern side of the ecliptic plane in spite of the source position in the southern hemisphere. Of course, we need to take effects of the deflection or highly non-radial propagation of the MC loop into consideration in such comparison. Wang et al. (2011) showed the deflection effects in many CMEs by a statistical study of their source locations. However, the required deflection looks too large in this case, 0.4 and $0.46 \mathrm{AU}$ for $\mathrm{Sb}-1$ and $\mathrm{Sb}-2$, respectively. As a result, two torus geometries La-1 and $L b-1$ remain as candidates for the model of the MC.

We further try to determine which model is taken as the optimum geometry for the observed $\mathrm{MC}$ by examining the accuracy of the fitting. Figure 5a shows the fitting result from the cylinder model (blue curve) and that from the torus model (red curve) for the adopted MC interval, 17/11:00-23:10. Figure 5b presents a similar comparison for the adopted interval, 17/13:05-23:20. In all four fittings, data points between two dot dash lines are disregarded. The most important difference between the torus and cylinder results is seen in the modeled $B x$ curve. It is being widely suspected that a cylindrical flux rope model with axis highly inclined from the ecliptic plane provides a prolonged southward $B z$. The point is, however, that such a flux rope yields $B x$ component, being not very small when traversed with large impact parameters, throughout the spacecraft passage, while the observed Bx values are close to 0 near the start and end times of the spacecraft crossing. It is seen that this observed feature can be reproduced by the toroidal flux rope model. It is thus concluded that a proper explanation of the 17 March $2016 \mathrm{MC}$ is provided by a toroidal model, but not by a cylinder model. It should be noted here that, for judgment of the successful fitting, we need to see the similarity between the observed and modeled field variations as well as the numerical estimate of the relative errors of fitting as defined by Additional file 1: Eq. (A1) in Appendix.

If the flux rope structure created in the corona extends to $1 \mathrm{AU}$ in a shape of a loop without significant change in the tilt of the loop plane, the resultant tilt of the loop should be close to that of the shear component of the solar field along the PIL. We estimated the orientation of the axial field of the flux rope loop near its apex for each of the modeled structures, following the expressions 

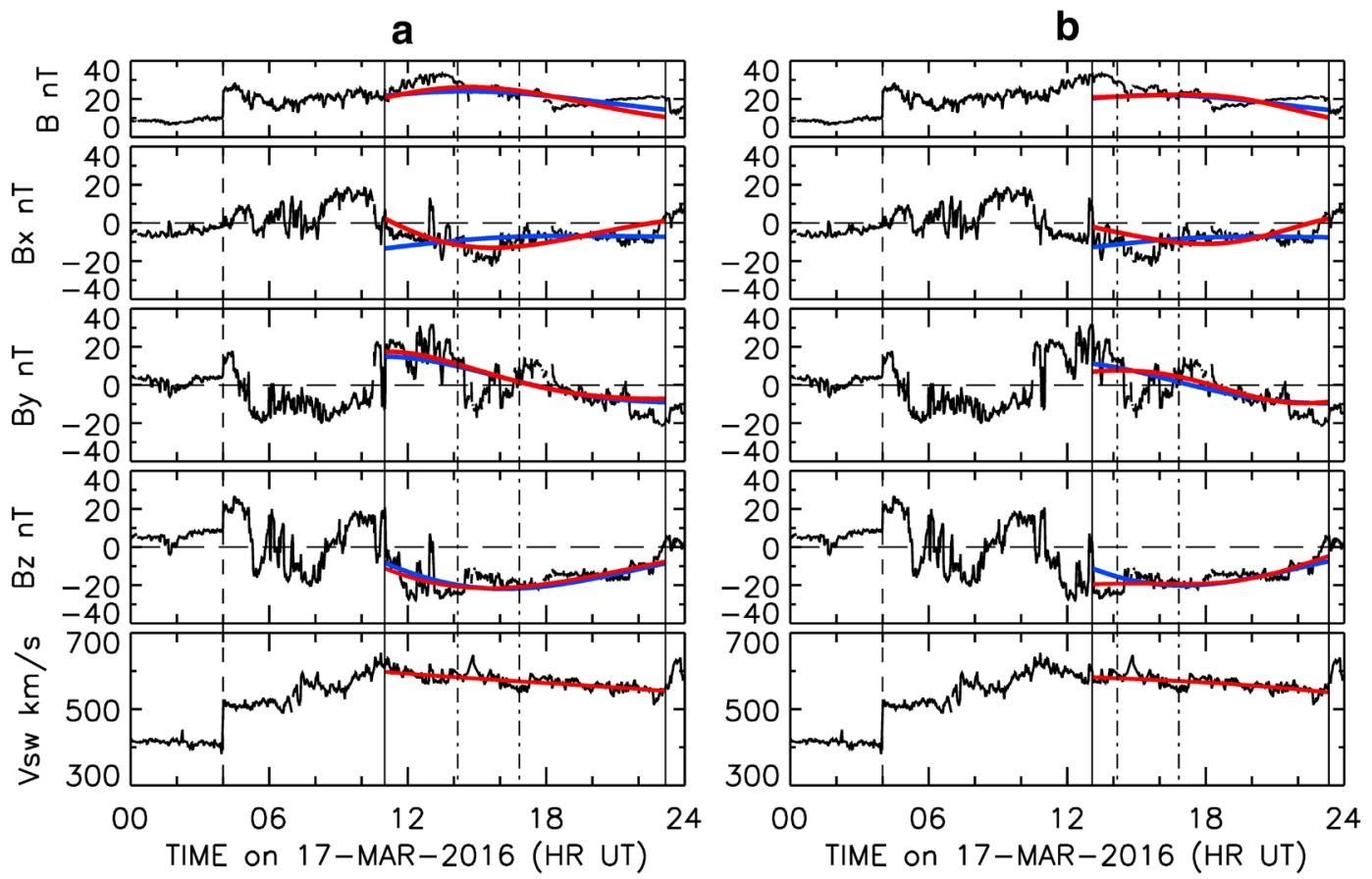

Fig. 5 Comparison of torus fit and cylinder fit for two MC intervals a 11:00-23:10 UT, and b 13:05-23:20 UT on 17 March 2015. Blue curves present the cylinder-fitted results, and red curves show the torus-fitted results

given by Marubashi et al. (2015). Figure 6 shows the result projected onto the $Y Z$ plane in GSE coordinates, where the red arrows show the orientation of axial filed near the apex of the loop for the four geometries obtained for the toroidal flux rope with right-handed chirality, and the blue arrows show the axial field orientation for the three flux ropes with left-handed chirality. For the cylindrical model, only the result from Lb-1 is shown (marked, CYL) because results from the four cylinder fits are all close to each other. We see that the axial field orientation is nearly parallel to the orientation of the sheared field in the solar source region only for the cases of the torus La-1 and the torus Lb-1. Although Kataoka et al. (2015) and Wang et al. (2016) selected the shorter interval for the MC, Kataoka et al. (2015) noticed that the field changes around the starting time of their $\mathrm{MC}$ selection are smooth and continuous, implying that the spacecraft encounter with the flux rope can be earlier. Therefore, we conclude here that the torus model Lb-1 provides the best interpretation of the March 17 2016 MC. It is true that we need to consider a possibility of significant rotation of the flux rope axis during propagating from the corona to $1 \mathrm{AU}$ when comparing the flux rope axis at $1 \mathrm{AU}$ with the direction of the PIL in the solar source region. However, many estimates drawn in Fig. 6 can be taken to be cautioning for careless determination of the flux rope axis as well.

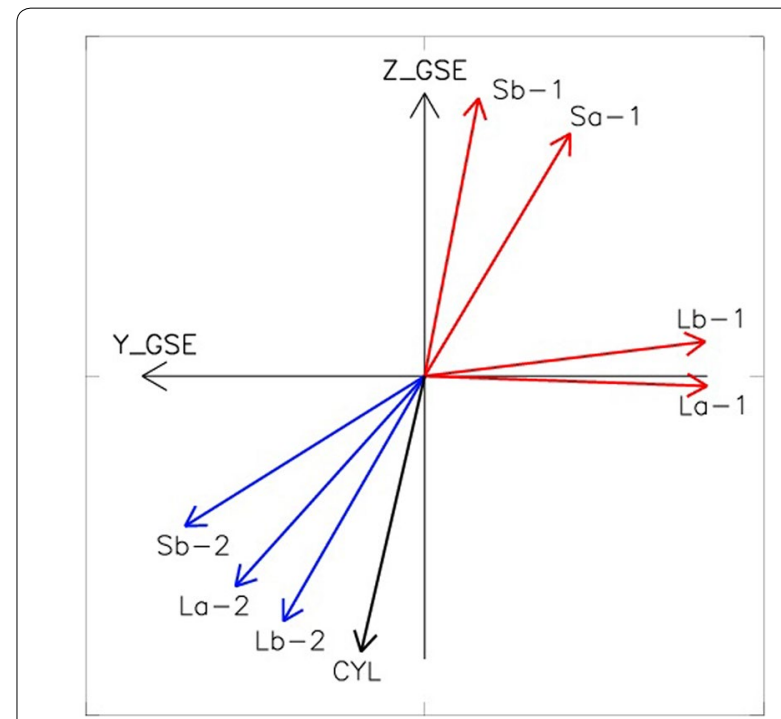

Fig. 6 Orientation in the $Y Z$ plane of the axial field estimated for each of the flux rope models. For toroidal flux rope models, cases of the right-handed and left-handed are shown by red and blue arrows. For cylindrical flux rope model, only one case is shown because the orientations are close to each other

Summarizing the above consideration, we conclude that the magnetic field variation shown in Fig. 1 is interpreted as follows. A flux rope structure of 
right-handed chirality is formed in association with the halo CME of 01:48 UT on 15 March 2015, from AR 12297, and it extends into interplanetary space making a flux rope loop with tilt of the loop plane nearly parallel to the PIL, or nearly parallel to the ecliptic plane in this case. Corresponding to the source position centered around S22W25, the WIND spacecraft crossed near the eastern flank, where the magnetic field is southward throughout the spacecraft passage, with $B x$ being negative toward the central region and $B y$ changing from positive to negative.

Finally, it is important to note in Additional file 1: Figure A1 that the same magnetic field variation can be reproduced by several different geometries in some cases. Such situations happen in cases where the spacecraft passed skimming the curved portion of a flux rope loop. An additional interesting feature of the 17 March MC is that observed magnetic fields were southward through the spacecraft passage, from the beginning to the end. Such a feature can be interpreted only by flux rope models with the curved shape. We can say that the observation of this $\mathrm{MC}$ is a kind of unusual happening, resulting from a peculiar spacecraft traverse through the $\mathrm{MC}$, which caused the prolonged southward $B z$.

\section{Ring current development}

We examine the storm evolution by estimating the injection rate of the ring current based on Burton's model (Burton et al. 1975; O'Brien and McPherron 2000). The model describes the ring current evolution with changes in solar wind characteristics in the form:

$$
\frac{\mathrm{d} D s t_{0}}{\mathrm{~d} t}=Q-\frac{D s t_{0}}{\tau}
$$

where $Q$ is the injection rate, $\tau$ is the decay time constant, and $D s t_{0}$ is the corrected Dst obtained by subtracting contribution of the magnetopause current as follows.

$$
D s t_{0}=D s t-7.26 \cdot \sqrt{P_{\mathrm{d}}}+11
$$

where $P_{\mathrm{d}}$ is the solar wind dynamic pressure. This expression means that we can estimate the ring current injection rate from the observed $D s t$ and solar wind data (for estimation of $P_{\mathrm{d}}$, if we assume that the decay time is constant. We write this quantity as:

$$
Q_{\text {obs }}=\frac{\mathrm{d} D s t_{0}}{\mathrm{~d} t}+\frac{D s t_{0}}{\tau}
$$

On the other hand, the injection rate should be expressed by a function of the solar wind characteristics, in more specifically by the dawn-to-dusk electric field in the solar wind $\left(E_{y}\right)$ as:

$$
Q_{\text {sw }}(t)=a+b \cdot S(t)
$$

$$
S(t)=\int_{0}^{t} F\left(t^{\prime}\right) \cdot E_{y}\left(t-t^{\prime}\right) \mathrm{d} t
$$

where $F(t)$ is the response function of the magnetosphere to the solar wind electric field, and normalized so that $\int F\left(t^{\prime}\right) \mathrm{d} t^{\prime}=1$.

In order to obtain the theoretical injection rate, $Q_{\mathrm{sw}}$, from the solar wind parameters, we need to determine the optimum response function $F(t)$ and the coefficients $a$ and $b$. For that purpose, we assume a simple form for the response function as depicted in Fig. 7, where $E_{y}$ is imposed at $t=0, T_{\mathrm{d}}$ is the delay time, $T_{\mathrm{r}}$ is the rising time, $T_{\mathrm{m}}$ is the main time, and $T_{\mathrm{f}}$ is the falling time. The optimum response function and the coefficients $a$ and $b$ are determined by cross-correlation analysis between $Q_{\text {obs }}(t)$ for an assumed $\tau$ and sets of $Q_{\text {sw }}(t)$ corresponding to many sets of $T_{\mathrm{d}}, T_{\mathrm{r}}, T_{\mathrm{m}}, T_{\mathrm{f}}$, and select a set which gives the highest correlation. We tried $T_{\mathrm{d}}=30,40,50$ min, $T_{\mathrm{r}}=10,20,30 \mathrm{~min}, T_{\mathrm{m}}=30,60,80,90 \mathrm{~min}$, and $T_{\mathrm{f}}=10,30,60,90 \mathrm{~min}$.

In Fig. 8, plotted are magnetic field intensity (nT), $B_{z}$ $(\mathrm{nT})$ in the GSM coordinate, $-V_{x}(\mathrm{~km} / \mathrm{s})$, dynamic pressure $\left(P_{\mathrm{d}}\right)$ in $\mathrm{nPa}, E_{y}$ in $\mathrm{mV} / \mathrm{m}, S(t)$ in $\mathrm{mV} / \mathrm{m}, D s t$ and $D s t_{0}$ (nT), and $Q_{\text {obs }}$ values corresponding to $\tau=4.0,6.0$, and $8.0 \mathrm{~h}$ (by solid, dotted, and dashed lies, respectively). Here, $S(t)$ shows the result for $\tau=4.0 \mathrm{~h}$ obtained by the method described above applied to the time interval from 06:00 UT on March 17 to 03:00 UT on March 18. The best correlation (coefficient $=0.882$ ) between $Q_{\text {obs }}(t)$ and $Q_{\mathrm{sw}}(t)$ was obtained for $T_{\mathrm{d}}, T_{\mathrm{r}}, T_{\mathrm{m}}, T_{\mathrm{f}}=40,10,90$, 30 min. $Q_{\text {sw }}(t)$ can be calculated by Eq (5) with $a=-26.3$ and $b=5.67$ (see Table 1). The delay time of $40 \mathrm{~min}$ is close to the transit time of the solar wind from the position of WIND to the magnetosphere. It is clear that any

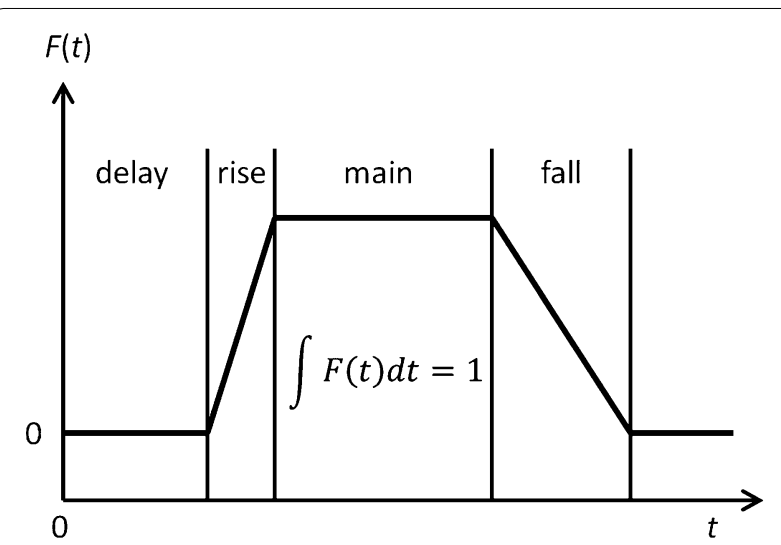

Fig. 7 Form of response function we assumed to evaluate the effect of the solar wind electric field on the injection rate of ring current. The lengths of four phases $\left(T_{d}, T_{r}, T_{m}, T_{f}\right)$ determine the injection rate 


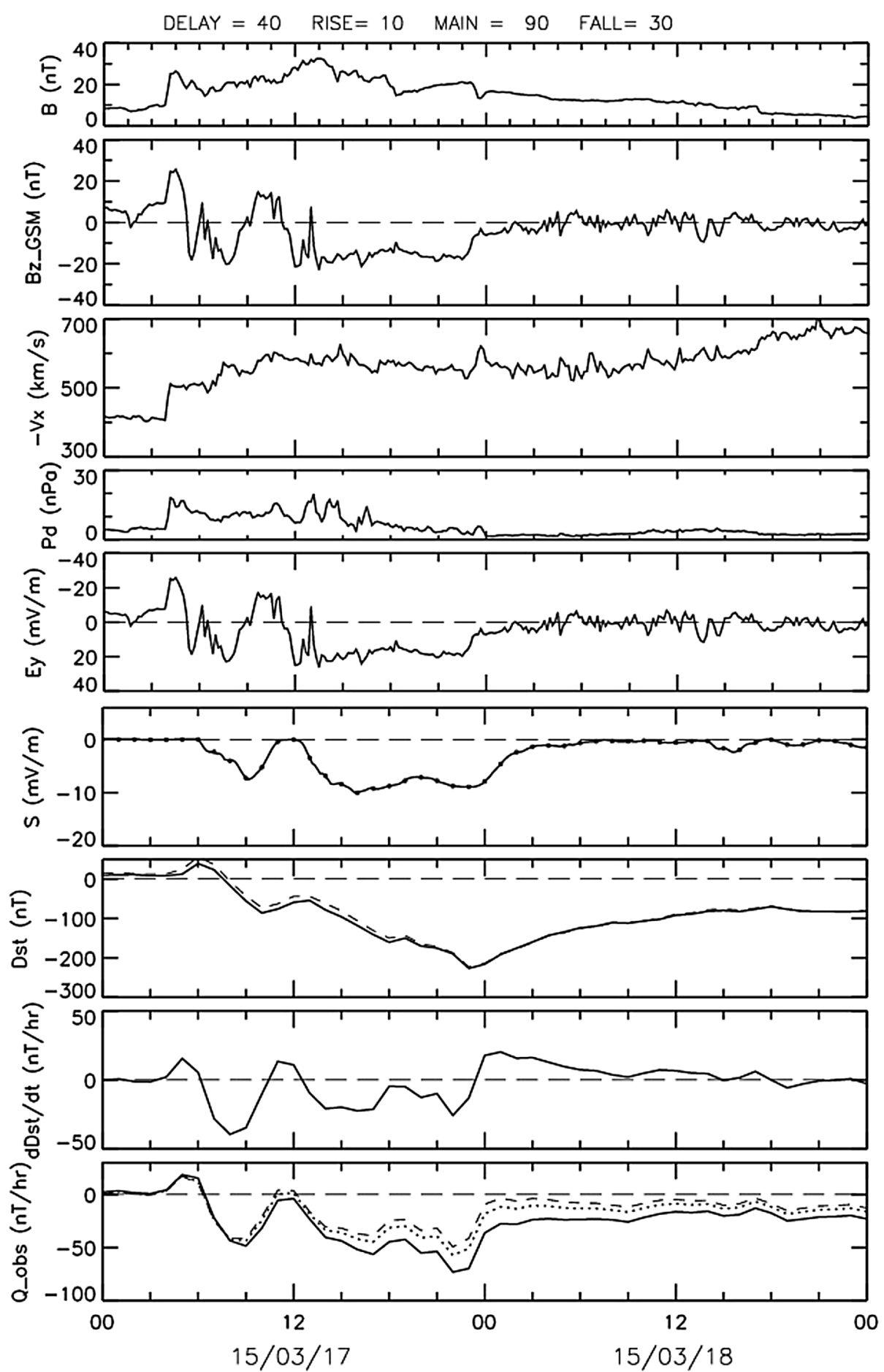

Fig. 8 Solar wind conditions and geomagnetic response for 17 March storm. Plotted are magnetic field intensity (B), BZ component in GSM coordinates, solar wind $V x$, dynamic pressure $\left(P_{\mathrm{d}}\right)$, solar wind electric field $\left(E_{y}=B z \times V x\right)$, the convolution $S$ defined by Eq. (5), the observed Dst (dashed line) and the modified Dst (solid line), the time derivative of modified Dst, and $Q_{\mathrm{obs}}$ defined by Eq. (3), where values for $\tau=4.0,6.0$, and 8.0 $\mathrm{h}$ are plotted by solid, dotted and dashed lines, respectively. Note the similarity of $S$ curve to $Q_{\mathrm{obs}}$ for $\tau=4.0$, and the difference between $S$ and $E_{\mathrm{y}}$. The $S$ curve shown here is for the parameter set, for $T_{\mathrm{d}}, T_{\mathrm{r}}, T_{\mathrm{m}}, T_{\mathrm{f}}=40,10,90,30 \mathrm{~min}$ 
Table 1 Optimum parameter sets for response and corresponding correlations

\begin{tabular}{llllllll}
\hline Time interval date/UT (start-end) & Delay $(\mathbf{m i n})$. & Rise $(\mathbf{m i n})$. & Main $(\mathbf{m i n})$. & Fall $(\mathbf{m i n})$. & Corr. coeff & Regress coeff_a & Regress coeff_b \\
\hline 17/06:00-18/03:00 & 40 & 10 & 90 & 30 & 0.882 & -26.3 & 5.67 \\
17/06:00-17/12:00 & 40 & 10 & 90 & 30 & 0.911 & 1.5 & 7.41 \\
17/12:00-17/19:00 & 40 & 10 & 90 & 30 & 0.984 & -19.8 & 4.98 \\
17/19:00-18/03:00 & 40 & 10 & 30 & 10 & 0.895 & -85.9 & 4.88 \\
\hline
\end{tabular}

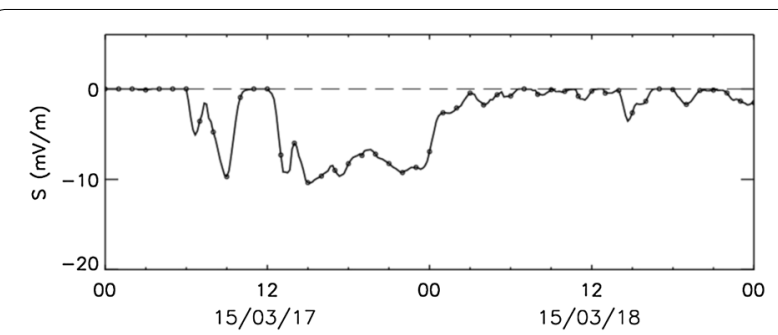

Fig. 9 An $S$ curve calculated for shorter $T_{m}\left(T_{m}=30 \mathrm{~min}\right)$. It reflects more closely the change in $E_{y}$

linear function of $E_{y}$ cannot reproduce the $Q_{\text {obs }}$ curve so well as the $S(t)$ curve. For the purpose of reference, Fig. 9 shows an $S(t)$ curve for $T_{\mathrm{m}}=30 \mathrm{~min}$. It is seen that the $S(t)$ curve becomes closer to the $E_{y}$ curve for this shorter main integration time.

Now that the injection rate is obtained from the solar wind observations, we can calculate the ring current development from Eq. (1), the solution of which is given by

$$
D s t_{0}(t)=\mathrm{e}^{-t / \tau} \cdot\left[D s t_{0}(0)+\int_{0}^{t} Q_{\text {sw }} \cdot \mathrm{e}^{t / \tau} \mathrm{d} t\right]
$$

Figure 10 shows the Dst values obtained from this expression and with correction for the magnetopause current in a black broken line together with the observed Dst

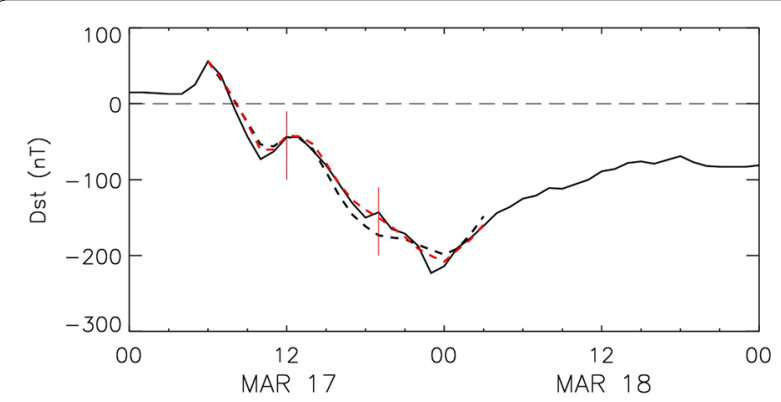

Fig. 10 Observed (solid line) and two modeled Dst curves. The black dashed line is the result of analysis which was done for the whole interval at once. The red dashed line shows the result from the analysis performed for three separate intervals (dashed line). We see general agreement between these two curves.

Another interesting feature in Fig. 8 is that three dents are evident in the $Q_{\text {obs }}$ curve (and also in the $S(t)$ curve) corresponding to the multi-step development of the storm. The first dent is apparently related to southward fields in the sheath after the shock. We examined the injection rate for each of the three dents separately. The result is listed in Table 1 for each of the three time intervals, together with the result from the correlation analysis for the whole interval of the storm main phase. Here, the results only for the case of decay constant of $4 \mathrm{~h}$ are shown because this case gives the best correlation. We obtained the same values for the parameter sets defining the response function for the first two intervals as those for the whole interval. It is also found that the coefficient $b$ for the first interval is $50 \%$ larger than the coefficient $b$ for the second interval. This means that the development of ring current was more strongly affected by the solar wind conditions in the first interval. The difference can be ascribed to the difference of solar wind density as pointed out by Kataoka et al. (2015) on the basis of Keika et al. (2015). This suggests that similar calculation of $D s t$ values from the analysis for separated intervals should yield better agreement with the observed values. In fact, the modeled $D s t$ in this way is shown in Fig. 10 by the red dashed line, which is much improved in reproducing the observed $D$ st.

Generally, geomagnetic responses to the solar wind conditions should change with time for other reasons. For example, Ohtani et al. (2007) pointed out the possible contribution of the tail current to Dst, implying that our present model should be too much simplified. Nevertheless, the result we obtained supports the basic correctness of the idea that the solar wind control of Dst development is described by the convolution such as Eqs. (5) and (6), though further refinements are desirable based on time-varying magnetospheric conditions.

\section{Conclusions}

We studied the 17 March 2015 storm focusing on the MC structure associated with the storm and on the storm development as geomagnetic response to the solar wind conditions. Our conclusions are summarized as follows. 
1. The magnetic field variation observed from 11:00 to $23: 10$ UT on 17 March 2015 is interpreted by a toroidal flux rope structure of right-handed chirality with the torus plane nearly parallel to the ecliptic plane. The observed variation is well reproduced by the model field for the spacecraft passage through the east side flank of the flux rope loop.

2. The above interpretation is consistent with the solar source event, a halo CME associated with a C9.1 flare which started at 01:15 UT on 15 March around S22W25. The consistency includes the resemblance between the magnetic structure of the $\mathrm{MC}$ and the magnetic structure around the solar source region, the geometrical relation between the MC axis and the PIL orientation, and the spacecraft crossing near the east flank of the MC corresponding to the solar source position in the western hemisphere.

3. It can be said that the geometry of the spacecraft crossing through the $\mathrm{MC}$ is a rather rare example in that the observed magnetic fields were southward throughout the passage. This feature can be reproduced only by a flux rope model with curved shape.

4. The observed Dst variation is explained as a geomagnetic response to the observed solar wind conditions by a newly developed expression for the injection rate of the storm ring current. The response is expressed by a convolution of the dawn-to-dusk electric field and some response function and not by the electric field itself.

\section{Additional file}

Additional file 1: Appendix. Outline of model-fitting for 17 March 2015 magnetic cloud. Table A1. Results of fitting with a cylinder model. Table A2. Results of fitting with a torus model. Figure A1. Drawings of seven torus geometries listed in Table A2 projected onto the YZ plane.

\section{Authors' contributions}

KM created the overall structure of the article and was responsible for drafting the full manuscript. KSC, RSK, SK and SHP collaborated with KM in discussing the contents and contributed their idea, especially on interpretation of solar images. HI contributed to the ring current analysis. All authors read and approved the final manuscript.

\section{Author details}

${ }^{1}$ Korea Astronomy and Space Science Institute, Daejeon 305-348, Korea. ${ }^{2}$ Asia Space Environment Research Consortium, Tokyo 1822-0024, Japan. ${ }^{3}$ National Institute of Information and Communications Technology, Tokyo 184-8795, Japan. ${ }^{4}$ University of Science and Technology, Daejeon 305-330, Korea. ${ }^{5}$ Trinity College Dublin, College Green, Dublin 2, Ireland.

\section{Acknowledgements}

Main part of the work was done when KM stayed at Korea Astronomy and Space Institute as a visiting scientist. We thank the WIND, SOHO, and SDO teams for providing the data online. We also acknowledge NASA's Space Physics Data Facility and the CDAW Data Center for our free use of appropriate data. This work also benefited from the SCOSTEP-WDS Workshop on Global Data Systems for the Study of Solar-Terrestrial Variability, held on 28-30 September 2015 in Tokyo.

\section{Competing interests}

The authors declare that they have no competing interests.

Received: 17 May 2016 Accepted: 22 October 2016

Published online: 08 November 2016

\section{References}

Burton RK, McPherron RL, Russell CT (1975) An empirical relationship between interplanetary conditions and Dst. J Geophys Res 80:4204-4214. doi:10.1029/JA080i031p04204

Cho K-S, Marubashi K, Kim R-S, Park S-H, Lim E-K (2016) Case studies on connection of CME and interplanetary conditions: September 2014 and March 2015 events. J Geophys Res (submitted)

Gopalswamy N, Akiyama S, Yashiro S, Xie H, Mäkelä P, Michalek G (2015) The mild space weather in solar cycle 24. In: Proceedings of the 14th international ionospheric effects symposium, pp 1-8

Gosling JT (1990) Coronal mass ejections and magnetic flux ropes in interplanetary space. In: Russell CT, Priest ER, Lee LC (eds) Physics of magnetic flux ropes. AGU, Washington, DC. Geophys Monogr Ser 58:343-364

Gosling JT, Teh W-L, Eriksson S (2010) A torsional Alfvén embedded within a small magnetic flux rope in the solar wind. Astrophys Lett 719:L36-L40. doi:10.1088/2041-8205/719/1/L36

Jian L, Russell CT, Luhmann JG, Skoug RM (2006) Properties of interplanetary cronal mass ejections at one AU during 1995-2004. Sol Phys 239:393436. doi:10.1007/s11207-006-0133-2

Kamide Y, Kusano K (2015) No major solar flares but the largest geomagnetic storm in the present solar cycle. Space Weather 13:365-367. doi:10.1002 /2015SW001213

Kataoka R, Shiota D, Kilpua E, Keika K (2015) Pileup accident hypothesis of magnetic storm on 17 March 2015. Geophys Res Lett 42:5155-5161. doi:1 0.1002/2015GL064816

Keika K, Ebihara Y, Kataoka R (2015) What caused the rapid recovery of the Carrington storm? Earth Planets Space 67:65. doi:10.1186/ s40623-015-0234-y

Kusano K, Bamba Y, Yamamoto TT, lida Y, Toriumi S, Asai A (2012) Magnetic field structures triggering solar flares and coronal mass ejections. Astrophys J 760:31. doi:10.1088/0004-637X/760/1/31

Liu YD, Huidong H, Wang R, Yang Z, Zhu B, Liu YA, Luhmann JG, Richardson JD (2015) Plasma and magnetic field characteristics of solar coronal mass ejections in relation to geomagnetic storm intensity and variability. Astrophys J Lett 809:L34. doi:10.1088/2041-8205/809/2/L34

Lopez R (1987) Solar cycle invariance in solar wind proton temperature relationships. J Geophys Res 92:11189-11194. doi:10.1029/ JA092iA10p11189

Marubashi K, Cho KS (2015) Non-uniqueness of the geometry of interplanetary magnetic flux ropes obtained from model-fitting. Sun Geosph 10:119-125

Marubashi K, Lepping R (2007) Long-duration magnetic clouds: a comparison of analyses using torus- and cylinder-shaped flux rope models. Ann Geophys 25:2453-2477. doi:10.5194/angeo-25-2453-2007

Marubashi K, Akiyama S, Yashiro S, Gopalswamy N, Cho KS, Park YD (2015) Geometrical Relationship between interplanetary flux ropes and their solar sources. Sol Phys 290:1371-1397. doi:10.1007/ s11207-015-0681-4

Neugebauer M, Gosling JT (1997) Particle and field signatures of coronal mass ejections in the solar wind. In: Crooker N, Joselyn JA, Feynman J (eds) Coronal mass ejections. AGU, Washington, DC. Geophys Monogr Ser 99:245-251

O'Brien TP, McPherron RL (2000) An empirical phase space analysis of ring current dynamics: solar wind control of injection and decay. J Geophys Res 105:7707-7719. doi:10.1029/1998JA000437

Ohtani S, Ebihara Y, Singer HJ (2007) Storm-time magnetic configurations at geosynchronous orbit: comparison between the main and recovery phases. J Geophys Res 112:A05202. doi:10.1029/2006JA011959

Sonnerup BUO, Cahill LJ Jr (1967) Magnetopause structure and attitude from Explorer 12 observations. J Geophys Res 72:171-183. doi:10.1029/ JZ072i001p00171 
Wang Y, Chen C, Gui B, Shen C, Ye P, Wang S (2011) Statistical study of coronal mass ejection source locations: understanding CMEs viewed in coronagraphs. J Geophys Res 116:A04104. doi:10.1029/20 10JA016101
Wang Y, Zhang Q, Liu J, Shen C, Shen F, Yang Z, Zic T, Vrsnak B, Webb DF, Liu R, Wang S, Zhang J, Hu Q, Zhuang B (2016) On the propagation of geoeffective coronal mass ejection during March 15-17, 2015. J Geophys Res 121:7423-7434. doi:10.1002/2016JA022924

\section{Submit your manuscript to a SpringerOpen ${ }^{\odot}$} journal and benefit from:

- Convenient online submission

- Rigorous peer review

Immediate publication on acceptance

- Open access: articles freely available online

- High visibility within the field

- Retaining the copyright to your article

Submit your next manuscript at springeropen.com 